Letter

\title{
miR-21 Promotes Keratinocyte Migration and Re-epithelialization During Wound Healing
}

\author{
Xue Yang1,2, Jun Wang², Shui-Long Guo², Kai-Ji Fan², Jun $\mathrm{Li}^{2}$, You-Liang Wang², Yan Teng ${ }^{\circledR}{ }^{\bowtie}$, and Xiao \\ Yang $1,2 \bowtie$
}

1. Model Organism Division, E-institutes of Shanghai Universities, Shanghai Jiao Tong University, P.R. China

2. State Key Laboratory of Proteomics, Genetic Laboratory of Development and Diseases, Institute of Biotechnology, Beijing, P.R. China

$\triangle$ Corresponding author: Xiao Yang, Ph.D., Institute of Biotechnology, 20 Dongdajie, Beijing 100071, P.R. China. Tel/Fax: 86-10-63895937(O); E-mail: yangx@nic.bmi.ac.cn. Or to: Yan Teng, Ph.D., Institute of Biotechnology, 20 Dongdajie, Beijing 100071, P.R. China. E-mail: tengyan0919@tom.com

(C) Ivyspring International Publisher. This is an open-access article distributed under the terms of the Creative Commons License (http://creativecommons.org/ licenses/by-nc-nd/3.0/). Reproduction is permitted for personal, noncommercial use, provided that the article is in whole, unmodified, and properly cited.

Received: 2011.01.24; Accepted: 2011.05.01; Published: 2011.05.30

\begin{abstract}
MicroRNAs involved in keratinocyte migration and wound healing are largely unknown. Here, we revealed the indispensable role of miR-21 in keratinocyte migration and in re-epithelialization during wound healing in mice. In HaCaT cell, miR-21 could be upregulated by TGF- $\beta 1$. Similar to the effect of TGF- $\beta 1$, miR- 21 overexpression promoted keratinocyte migration. Conversely, miR-21 knockdown attenuated TGF- $\beta 1$-induced keratinocyte migration, suggesting that miR-21 was essential for TGF- $\beta$-driven keratinocyte migration. Furthermore, we found that miR-21 was upregulated during wound healing, coincident with the temporal expression pattern of TGF- $\beta 1$. Consistently, knockdown of endogenous miR-21 using a specific antagomir dramatically delayed re-epithelialization possibly due to the reduced keratinocyte migration. TIMP3 and TIAM1, direct targets of miR-21, were verified to be regulated by miR-21 in vitro and in vivo, indicating that these two molecules might contribute to miR-21-induced keratinocyte migration. Taken together, our results demonstrate that miR-21 promotes keratinocyte migration and boosts re-epithelialization during skin wound healing.
\end{abstract}

Key words: miR-21, keratinocyte, migration, wound healing

\section{Introduction}

Cutaneous wound healing is a highly orchestrated multi-step process. An essential feature of a healed wound is re-epithelialization which relies on two basic functions of keratinocytes: proliferation and migration [1]. The re-epithelialization process is influenced by a combination of growth factors and cytokines including transforming growth factor- $\beta$ (TGF- $\beta$ ) [2]. During wound healing, TGF- $\beta 1$ expression is significantly increased, and keratinocytes are the major target cells of TGF- $\beta 1$ [3]. In vitro, the effects of TGF- $\beta$ signaling are paradoxical: keratinocyte migration is enhanced whereas keratinocyte prolifera- tion is inhibited [4]. Genetic ablation of TGF- $\beta 1$, its receptors and its downstream mediators, Smads, have been reported to either reduce or enhance the rate of wound repair [5-7]. Therefore, identifying the downstream effectors of TGF- $\beta$ signaling involved in this process was pivotal for better understanding of the molecular mechanism underlying the cellular response to TGF- $\beta$ signaling during wound healing.

MicroRNAs (miRNAs) are small, single-stranded, noncoding RNAs, which usually bind to the 3'-untranslated region of target mRNAs and are capable of inducing posttranscriptional gene regula- 
tion by blocking translation or by degrading the target mRNA [8]. Several lines of evidence have shown that miRNAs regulate keratinocyte proliferation and migration $[9,10]$. miR-205 promotes epidermal keratinocyte migration through targeting SHIP2 [9]. In a mouse model of ischemic wound, HIF-1a-induced miR-210 attenuates keratinocyte proliferation by targeting E2F3 [10]. However, other miRNAs involved in modulating keratinocyte proliferation and migration remain to be fully defined.

miR-21, the most frequently upregulated miRNA in human cancers and other diseases, plays important roles in cell proliferation, differentiation, apoptosis, epithelial to mesenchymal transition (EMT) and migration through its distinct targets [11-13]. For instance, miR-21 promotes migration in glioma cell by targeting RECK and TIMP3 [11], and in colon cancer cell by targeting TIAM1 [12]. Previous studies have shown that miR-21 is highly expressed in epidermis and hair follicle [14], and is aberrantly elevated in psoriatic epidermis [15]. However, the function of miR-21 in keratinocyte and cutaneous wound healing is still underdetermined.

In the current study, we revealed an important role of miR-21 in keratinocyte migration as well as during wound healing.

\section{Results and Discussion}

Previous studies have shown that TGF- $\beta 1$ promotes expression of mature miR-21 through post-transcriptional mechanism in human vascular smooth muscle cell and colon cancer cell $[12,16]$. To determine whether TGF- $\beta 1$ could regulated miR-21 expression in keratinocyte, HaCaT, a human keratinocyte cell line, was treated with TGF- $\beta 1$. miR-21 expression was dramatically increased at 6 hours after TGF- $\beta 1$ treatment (Figure 1A). Previous studies have revealed that miR-21 plays important roles in regulating cell proliferation in various cell types [13]. However, overexpression or knockdown of miR-21 did not affect the proliferation of $\mathrm{HaCaT}$ cell (Figure 1B and Supplementary Material: Figure S1).

We next checked whether miR-21 affected keratinocyte migration. Scratch wound-healing assay was performed to examine keratinocyte migration. Consistent with the previous work [17], TGF- $\beta 1$ remarkably enhanced keratinocyte migration by $48 \%$ at 12 hours (Figure 1C, P<0.01). Similarly, migration distance of miR-21-transfected HaCaT cells was significantly increased by $62 \%$ at 12 hours compared with that of scrambled-miRNA-transfected cells (Figure $1 \mathrm{C}, \mathrm{P}<0.01$ ). The above results led us to speculate that miR-21 might be responsible for TGF- $\beta$-driven keratinocyte migration. We engineered miR-21 with antisense oligonucleotides (miR-21 AS) to knockdown the upregulated expression of miR-21 induced by TGF- $\beta 1$ (Figure 1D). As expected, the enhanced migration caused by TGF- $\beta 1$ was dramatically attenuated by miR-21 knockdown (Figure 1D, P<0.01). These data suggested that TGF- $\beta 1$ could promote keratinocyte migration through upregulating miR-21 expression in HaCaT cell.

Previous studies have identified some targets of miR-21 responsible for cell migration [11-12, 18]. Tissue inhibitor of metalloproteinase-3 (TIMP3) is capable of inhibiting migration in glioma cell and in breast cancer cell [11, 18], and is downregulated during wound healing [19] and in psoriatic epidermis [20]. We determined TIMP3 expression in HaCaT cells transfected with miR-21 mimic or scrambled-miRNA. Real-time PCR results revealed that TIMP3 was markedly reduced by $38 \%$ in miR-21-tansfected HaCaT cells compared with that in scrambled-miRNA-transfected control cells (Figure 1E, $\mathrm{P}<0.01$ ). To further determine whether miR-21 could directly regulate TIMP3, full length of TIMP3 mRNA 3'UTR was inserted to the downstream of luciferase open reading frame in pGL3-CM reporter vector. When introduced into HaCaT cells, the TIMP3 3'UTR reporter showed a $60 \%$ reduction in luciferase activity in the miR-21-transfected cells compared with that in scrambled-miRNA-transfected cells (Figure 1F, $\mathrm{P}<0.01$ ). Previous evidence revealed that TIAM1 (T-lymphoma invasion and metastasis inducing protein), a direct target of miR-21, is required for miR-21 to enhance migration and invasion in colon cancer cell [12]. Real-time PCR results confirmed that miR-21 overexpression with mimic caused a $25 \%$ reduction of TIAM1 expression in HaCaT cell (Figure 1G, P<0.01). The above data implied that TIMP3 and TIAM1 might be responsible for miR-21-promoted keratinocyte migration.

To investigate the function of miR-21 in vivo, we examined miR-21 expression during wound healing by Northern blot. 4-mm full-thickness punch-biopsy wounds were made on the back of mice. Coincident with the temporal upregulation of TGF- $\beta 1$, miR-21 expression at wound edge showed 2.8-3.9 fold increases after injury (Figure 2A). To investigate the functional consequences of miR-21 upregulation at wound edge, we downregulated endogenous miR-21 expression by administering Pluonic gel containing miR-21 AS to wounded skin. Northern blot result showed that miR-21 was effectively decreased by $44 \%$ at wound edge treated with miR-21 AS compared with that with scrambled AS 3 days after injury (Figure $2 \mathrm{~B})$. 
A

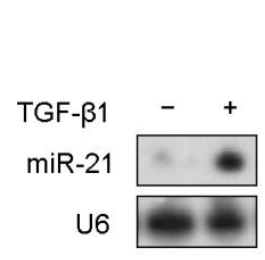

C
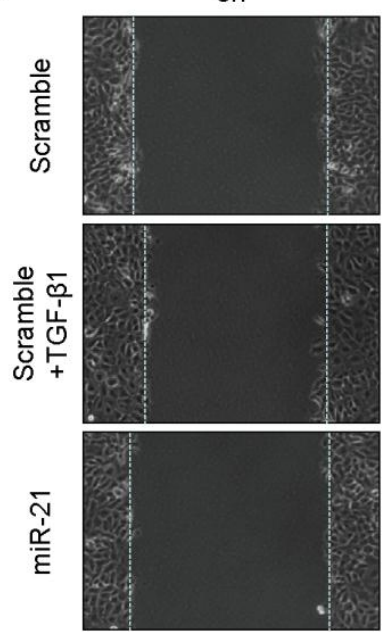

D
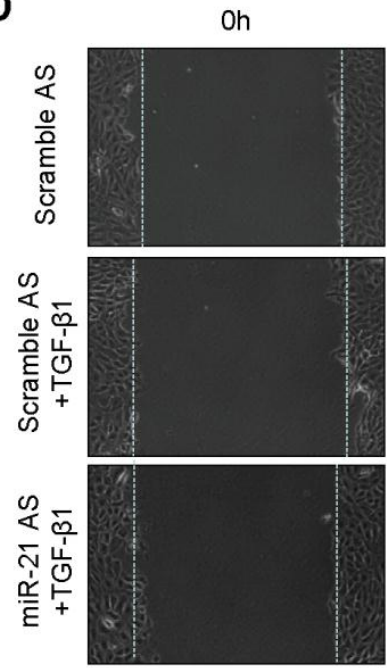

E

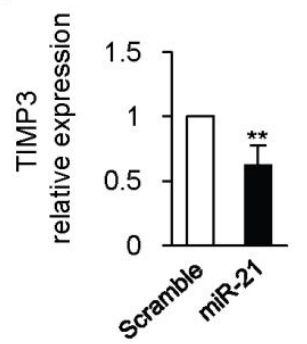

B

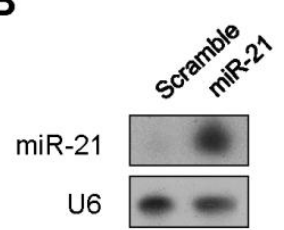

$12 \mathrm{~h}$
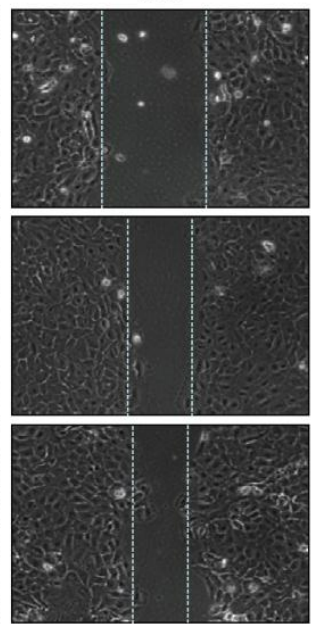

$24 \mathrm{~h}$
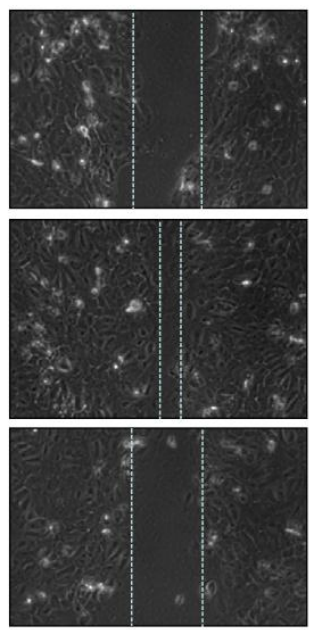

$\mathbf{F}$

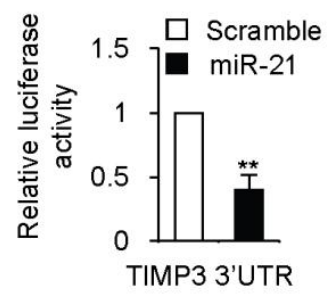

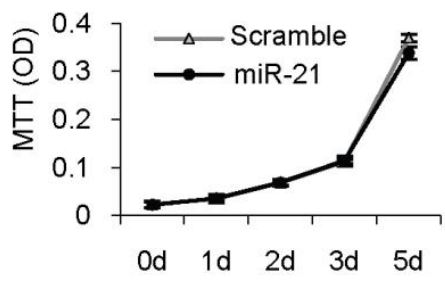
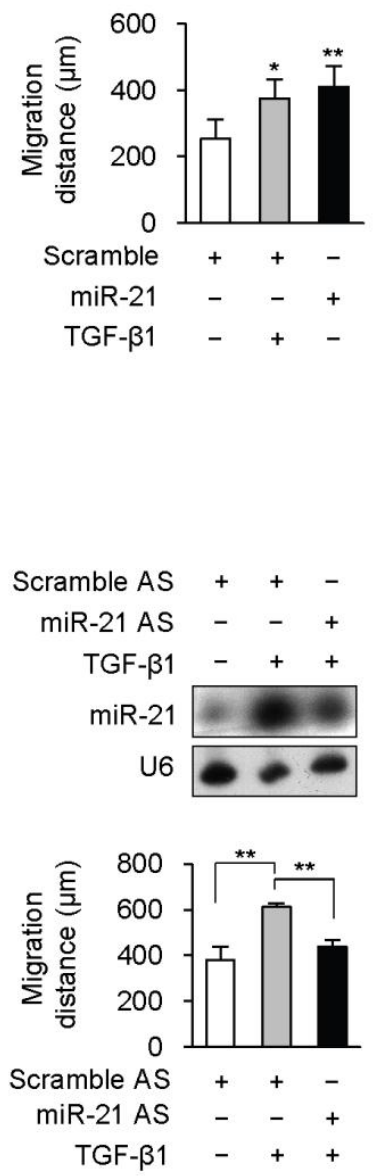

G

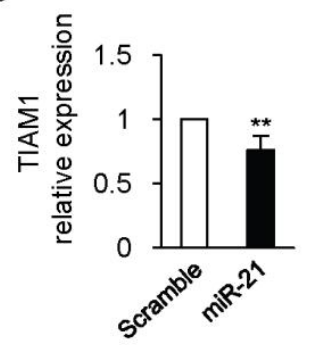

Figure 1: TGF-B1 upregulated miR-21 expression to promote keratinocyte migration in HaCaT cell. (A), miR-21 expression was detected by Northern blot in HaCaT cell treated with or without TGF-B1. (B), MTT assay showed that there was no obvious difference between miR-21-tansfected HaCaT cells (miR-21) and scrambled-miRNA-transfected (Scramble) cells. miR-21 overexpression with mimic were confirmed by Northern Blot. (C), miR-21 promoted cell migration in $\mathrm{HaCaT}$ cell. Scratch wound-healing assay was conducted in miR-21-transfected HaCaT cells, and control 
cells with or without TGF-B1 treatment. Migration distance was measured at 0,12 hours after cells were scratched. (D), The enhanced migration induced by TGF-B1 was dramatically blocked by miR-21 AS, but not by Scramble AS. Northern blot confirmed that the increased expression of miR-21 induced by TGF-B1 was downregulated by miR-21 AS. Migration distance was measured at 0, 24 hours after cells were scratched. (E), Real-time PCR analysis revealed that miR-21 overexpression with miR-21 mimic suppressed TIMP3 expression. (F), Luciferase activities were measured in $\mathrm{HaCaT}$ cells cotransfected with the reporter constructs containing 3'UTR of TIMP3 and miR-21 mimic or Scramble miRNA. (G), Real-time PCR analysis revealed that miR-21 overexpression with miR-21 mimic suppressed TIAM1 expression. Values represent mean $\pm S D . n=5 .{ }^{*} \mathrm{P}<0.05 .{ }^{* *} \mathrm{P}<0.01$.

A

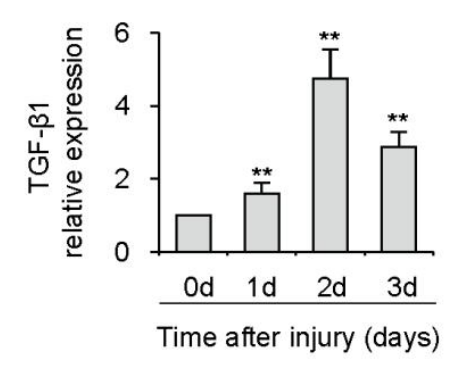

C

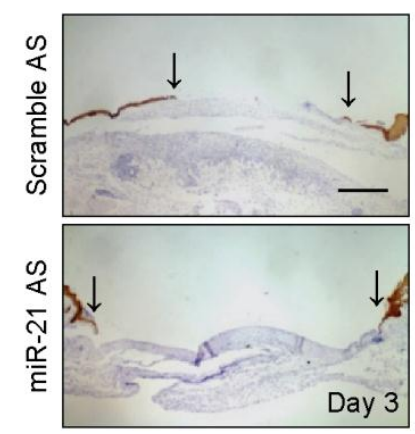

D

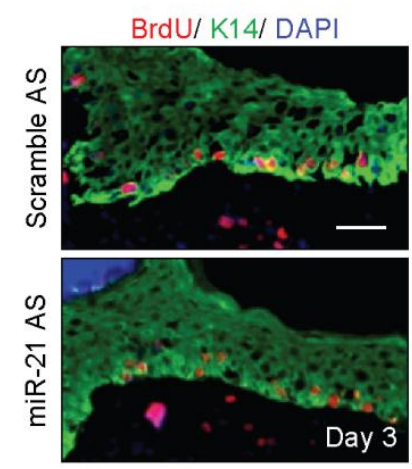

B

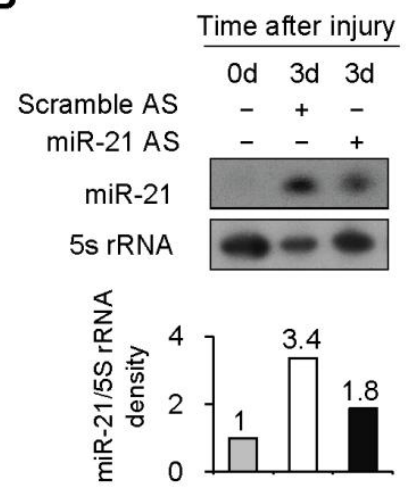

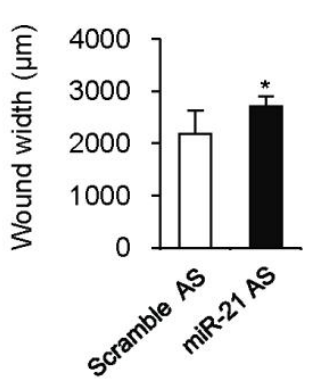

E

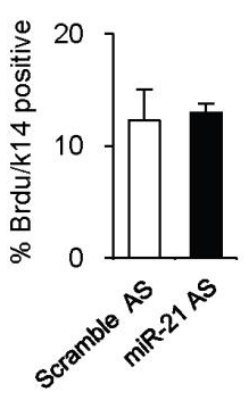

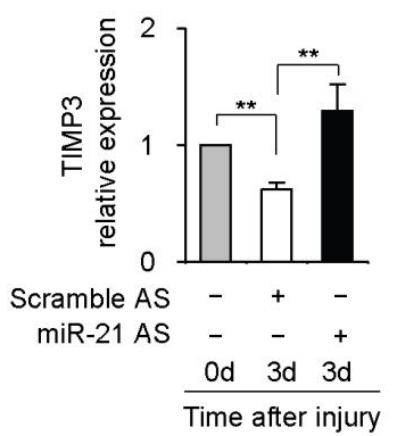

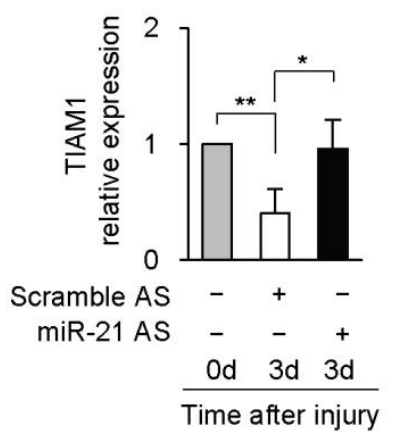

Figure 2: miR-21 promoted re-epithelialization during wound healing in mice. (A), Real-time PCR and Northern blot were performed to detect expression of TGF-B1 and miR-21 at wound edge at $0,1,2$ and 3 days after injury. (B), Northern blot result showed that miR-21 at wound edge treated with miR-21 AS was effectively decreased by $44 \%$ compared with that at control wound edge at 3 days after injury. (C), Quantification of wound widths over 3 days after injury. Sections of wounded skin with miR-21 AS or Scramble AS treatment was immunostained with K14 antibody (brown) at 3 days after injury. Arrows indicated the leading edges of wounded epidermis. $n=5$. (D), Immunofluorescence analysis with BrdU (red) and K14 (green) antibodies staining showed that no obvious difference was found in percentage of BrdU-positive keratinocytes in wounded skin treated with miR-21 AS or Scramble AS. $n=4$. $(E)$, Real-time PCR was performed to detect expression of TIMP3 and TIAM1 at wound edge with miR-21 AS or scramble AS at 3 days after injury. Values represent mean \pm SD. Scale bars: $400 \mu \mathrm{m}(C) ; 50 \mu \mathrm{m}(\mathrm{D}) .{ }^{*} \mathrm{P}<0.05 .{ }^{* *} \mathrm{P}<0.01$ 
Wounds were immunostained with Keratin 14 antibody for examination of re-epithelialization. We observed that the wound widths were larger and neo-epithelial lengths were shorter in healing skin treated with miR-21 AS compared with controls 3 days after injury (Figure $2 \mathrm{C}, \mathrm{n}=5, \mathrm{P}<0.05$ ), indicating that knockdown of endogenous miR-21 impaired re-epithelialization. Consistent with the effect of miR-21 on keratinocyte proliferation in vitro, we found that miR-21 downregulation did not affect keratinocyte proliferation at wound edge by BrdU incorporation assay (Figure 2D), suggesting that the delayed re-epithelialization caused by miR-21 downregulation was possibly due to the reduced keratinocyte migration. In addition, we found expression levels of TIMP3 and TIAM1 were decreased at control wound edge, but not at wound edge treated with miR-21 AS (Figure 2E, $\mathrm{P}<0.05$ ), indicating that miR-21 promoted keratinocyte migration possibly via inhibiting the expression of TIMP3 and TIAM1 in re-epithelialization process.

Previous studies have demonstrated that miR-21 promotes migration in some cell types including glioma cell [11] and colon cancer cell [12]. Here, we verified a similar role of miR-21 in keratinocyte migration. miR-21 overexpression with mimic dramatically enhanced keratinocyte migration. Moreover, for the first time, our results implicated that miR-21, as a downstream effector of TGF- $\beta 1$, was required for TGF- $\beta 1$-induced keratinocyte migration. miR-21 expression was dramatically increased after TGF- $\beta 1$ treatment in HaCaT cell. Similar to the effect of TGF- $\beta 1$, miR-21 overexpression with mimic could accelerate keratinocyte migration. Conversely, miR-21 knockdown blocked the TGF- $\beta 1$-dirven migration. Importantly, we further confirmed the role of miR-21 in wound healing in mice. miR-21 expression was significantly upregulated at wound edge, whose temporal expression pattern was coincident with that of TGF- $\beta 1$, suggesting that miR-21 upregulation at wound edge might be caused by TGF- $\beta 1$. When the upregulation of endogenous miR-21 was suppressed by miR-21 AS at wound site, re-epithelialization was markedly retarded. This delayed re-epithelialization was likely due to the decreased keratinocyte migration. Supportively, TIMP3 and TIAM1, direct targets of miR-21 [11, 12, 18], exhibited the inverse correlation with miR-21 expression in $\mathrm{HaCaT}$ cell and at wound edge of mouse, indicating that miR-21 might promote keratinocyte migration in vitro and wound healing in vivo via inhibiting the expression of TIMP3 and TIAM1. Other targets involved in this process remained to be further investigated.

\section{Conclusion}

Overall, our data revealed that miR-21 was indispensable for TGF- $\beta$-driven keratinocyte migration in vitro and could promote re-epithelialization during wound healing in mice.

\section{Materials and Experimental Design}

See Supplementary Material.

\section{Supplementary Material}

Material and Experimental Design/ Figure S1

http://www.biolsci.org/v07p0685s1.pdf.

\section{Ackowledgments}

This work was supported by grants 2011CB504200 and 2011CB901601 from Chinese National Key Program on Basic Research, grant 30700423 and 31030040 from National Natural Science Foundation of China, and grant 5082018 from Beijing Natural Science Foundation of China.

\section{Conflict of Interests}

The authors have declared that no conflict of interest exists.

\section{References}

1. Broughton G 2nd, Janis JE, and Attinger CE. The basic science of wound healing. Plast Reconstr Surg. 2006; 117 (Suppl 7): 12S-34S.

2. Amendt C, Mann A, Schirmacher P, et al. Resistance of keratinocytes to TGFbeta-mediated growth restriction and apoptosis induction accelerates re-epithelialization in skin wounds. J Cell Sci. 2002; 115(Pt 10):2189-98.

3. Werner S, Grose R. Regulation of wound healing by growth factors and cytokines. Physiol Rev. 2003; 83(3):835-70.

4. Räsänen K, Vaheri A. TGF-beta1 causes epithelial-mesenchymal transition in $\mathrm{HaCaT}$ derivatives, but induces expression of COX-2 and migration only in benign, not in malignant keratinocytes. J Dermatol Sci. 2010; 58(2):97-104.

5. Crowe MJ, Doetschman T, Greenhalgh DG. Delayed wound healing in immunodeficient TGF-beta 1 knockout mice. J Invest Dermatol. 2000; 115(1):3-11.

6. Owens P, Engelking E, Han G, et al. Epidermal Smad4 deletion results in aberrant wound healing. Am J Pathol. 2010; 176(1):122-33.

7. Grose $\mathrm{R}$ and Werner S. Wound-healing studies in transgenic and knockout mice. Mol Biotechnol. 2004; 28: 147-66.

8. Bartel DP. MicroRNAs: genomics, biogenesis, mechanism, and function. Cell. 2004; 116(2): 281-97.

9. Yu J, Peng H, Ruan Q, et al. MicroRNA-205 promotes keratinocyte migration via the lipid phosphatase SHIP2. FASEB J. 2010; 24(10):3950-9.

10. Biswas S, Roy S, Banerjee J, et al. Hypoxia inducible microRNA 210 attenuates keratinocyte proliferation and impairs closure in a murine model of ischemic wounds. Proc Natl Acad Sci U S A. 2010; 107: 6976-81.

11. Gabriely G, Wurdinger T, Kesari S, et al. MicroRNA 21 promotes glioma invasion by targeting matrix metalloproteinase regulators. Mol Cell Biol. 2008; 28(17):5369-80. 
12. Cottonham CL, Kaneko S, Xu L. miR-21 and miR-31 converge on TIAM1 to regulate migration and invasion of colon carcinoma cells. J Biol Chem. 2010; 285(46):35293-302.

13. Krichevsky AM, Gabriely G. miR-21: a small multi-faceted RNA. J Cell Mol Med. 2009;13(1):39-53.

14. Yi R, O'Carroll D, Pasolli HA, et al. Morphogenesis in skin is governed by discrete sets of differentially expressed microRNAs. Nat Genet. 2006; 38(3):356-62.

15. Gu X, Nylander E, Coates PJ, et al. Effect of Narrow-band Ultraviolet B Phototherapy on p63 and MicroRNA (miR-21 and miR-125b) Expression in Psoriatic Epidermis. Acta Derm Venereol. 2011; [Epub ahead of print]

16. Davis BN, Hilyard AC, Lagna G, et al. SMAD proteins control DROSHA-mediated microRNA maturation. Nature. 2008; 454(7200):56-61.

17. Hebda PA. Stimulatory effects of transforming growth factor-beta and epidermal growth factor on epidermal cell outgrowth from porcine skin explant cultures. J Invest Dermatol. 1988;91(5):440-5.

18. Song B, Wang C, Liu J, et al. MicroRNA-21 regulates breast cancer invasion partly by targeting tissue inhibitor of metalloproteinase 3 expression. J Exp Clin Cancer Res. 2010; 29:29.

19. Soo C, Shaw WW, Zhang X, et al. Differential expression of matrix metalloproteinases and their tissue-derived inhibitors in cutaneous wound repair. Plast Reconstr Surg. 2000; 105(2):638-47

20. Zibert JR, Løvendorf MB, Litman T, et al. MicroRNAs and potential target interactions in psoriasis. J Dermatol Sci. 2010; 58(3):177-85.

21. Guo SL, Peng Z, Yang X, et al. miR-148a Promoted Cell Proliferation by Targeting p27 in Gastric Cancer Cells. Int J Biol Sci. 2011;7(5):567-74.

22. Reynolds LE, Conti FJ, Silva R, et al. alpha3beta1 integrin-controlled Smad7 regulates reepithelialization during wound healing in mice. J Clin Invest. 2008; 118(3):965-74. 